\title{
Decentralized Event-Triggered Consensus for Linear Multi-Agent Systems under General Directed Graphs *
}

\author{
Dapeng Yang ${ }^{\mathrm{a}, \mathrm{b}}$, Wei Ren ${ }^{\mathrm{c}}$, Xiangdong Liu ${ }^{\mathrm{a}}$, Weisheng Chen ${ }^{\mathrm{d}}$

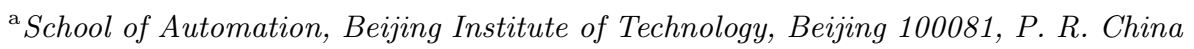 \\ ${ }^{\mathrm{b}}$ Beijing Urban Construction Design ES Development Group Co., Limited, Beijing 100037, P. R. China \\ ${ }^{\mathrm{c}}$ Department of Electrical and Computer Engineering, University of California, Riverside 92521, USA \\ ${ }^{\mathrm{d}}$ School of Aerospace Science and Technology, Xidian University, Xi'an 710071, P. R. China
}

\begin{abstract}
In this paper, the event-triggered consensus problem is studied for multi-agent systems with general linear dynamics under a general directed graph. Based on state feedback, we propose a decentralized event-triggered consensus controller (ETCC) for each agent to achieve consensus, without requiring continuous communication among agents. Each agent only needs to monitor its own state continuously to determine when to trigger an event and broadcast its states to its out-neighbors. The agent updates its controller when it broadcasts its states to its out-neighbors or receives new information from its in-neighbors. The ETCC can be implemented in multiple steps. it is proved that under the proposed ETCC there is no Zeno behavior exhibited. To relax the requirement of continuous monitoring of each agent's own states, we further propose a self-triggered consensus controller (STCC). Simulation results are given to illustrate the theoretical analysis and show the advantages of the event-triggered and self-triggered controllers proposed in this paper.
\end{abstract}

Key words: Decentralized event-triggering; consensus control; multi-agent systems; general linear dynamics; directed graphs.

\section{Introduction}

In the last decade, the consensus problem of continuous-time multi-agent systems (MAS) has been attracting much attention due to its wide applications. Many significant works have been obtained, e.g., see [8], [9], [11], [12], [16], and $[17]$, just to name a few. Note that in the above works the agents need to continuously employ their own and neighbors' states and hence these states need to be obtained continuously. To avoid this disadvantage, some researchers has begun to study the centralized/distributed event-triggered

\footnotetext{
^ This work was supported by National Natural Science Foundation of China (61433003, 60904003, 61174213, 61528301, 61120106010), the Program for New Century Excellent Talents in University (NCET-10-0665), and was partially supported by the National Science Foundation under grant ECCS-1307678. A preliminary version of this paper has been presented at the IEEE Conference on Decision and Control, Los Angles, CA, 2014. Corresponding author: Weisheng Chen.

Email addresses: yangdp1988@163.com (Dapeng Yang), ren@ee.ucr.edu (Wei Ren), xdliu@bit.edu.cn (Xiangdong Liu), wshchen@126. com (Weisheng Chen).
}

consensus problem. The event-triggered average-consensus problem was considered for MAS with single-integrator dynamics in [3] and [4]. The event-triggered consensus problem for MAS with general linear dynamics was investigated in [18]. Periodic event/self-triggered consensus for general linear MAS and distributed convex optimization problem based on event-triggered algorithms have been studied in [21] and [22]. While the controllers in [3], [4], and [18] are often updated less by using the event-triggered algorithms than using the time-triggered ones, they still require the agents to communicate with their neighbors continuously.

It is well known that unnecessary communication can lead to a waste of energy. Continuous communication would also cause the communication resource competition among agents. To remove the requirement for continuous communication and hence reduce the communication cost, researchers have begun to study the quantized consensus or the event-/self-triggered consensus. For example, the event triggering idea was used to solve the continuous-time quantized average consensus problem in [1] with the graph being weight-balanced and weakly-connected. Besides, periodic event-triggered consensus algorithm was studied in [10] for single-integrator agents over undirected connected communication topologies. A self-triggered control algo- 
rithm for single-integrator agents was given in [2]. In [14], a decentralized event-triggered consensus algorithm was considered for single- and double- integrator agents. To remove the requirement of global information or synchronous broadcasting as in sampled-data approaches, [7] studied the event-triggered control based on only local state errors. In addition, the consensus problem of double-integrator MAS with intermittent communications was investigated in [15]. Note that during the disconnected communicating time intervals, the communication is still continuous, but not discrete. An event-triggered distributed consensus optimization algorithm was proposed in [21]. However, in [1], [2], [7], [10], [14], [15] and [21], the agents were assumed to be with single- or double- integrator dynamics. For MAS with general linear dynamics, [6], [18], [19], and [20] have recently considered the event-triggered consensus problem. However, in [6] and [20] the final consensus error could only converge to a neighborhood of zero and [18], [19], and [20] required continuous communication of neighbors' states to check the triggering conditions. The communication topology in [19] was assumed to be undirected. Also [14] only considered the event-triggered consensus for double-integrator dynamics under undirected graphs. In short, the event-triggered consensus problem with zero final consensus error for general linear MAS without continuous communication under directed graphs has not been addressed.

Motivated by the above discussion, we consider the consensus problem for MAS with general linear dynamics under a general directed graph based on an event-triggered broadcasting scheme by expanding on our preliminary work reported in [22]. The communication topology among the agents is assumed to be a general directed graph containing a directed spanning tree. With state feedback, we first propose a decentralized event-triggered consensus controller (ETCC) implemented in multiple steps for each agent to achieve consensus. Under our proposed controller, there is no continuous communication required among agents. We further prove that there is no Zeno behavior exhibited during the control process, that is, the event would not be triggered continuously. Note that if the Zeno behavior cannot be avoided, then essentially continuous communication is required again. Note that under the ETCC, each agent needs to monitor its own state continuously. To relax this limitation, we further propose a self-triggered consensus controller (STCC), where the next triggering instant is preset by the agent itself at the previous triggering instant. Here the stability analysis and the exclusion of the Zeno behavior of the closed-loop systems are partly inspired by [9] and [14]. However, due to the significant challenges caused by the coupling of nonmonotonically changing measurement errors, general linear dynamics, and directed communication topologies, the convergence of the consensus errors and the non-Zeno analysis are more difficult than before. The primary contributions of the paper are summarized as follows.

(1) We solve the event-triggered/self-triggered consensus problem for multi-agent systems with general linear dynamics under general directed graphs without the need for continuous communication in either controller update or triggering condition monitoring. In addition, our results guarantee zero final consensus error. Most works in the existing literature have some limitations such as agents' dynamics, communication topologies, nonzero final consensus error, and continuous communication. So, the methods proposed in the literature cannot be directly used in our paper.

(2) By using the matrix exponential function $e^{A t}$, a piecewise continuous control input is designed in the proposed ETCC to estimate the current states of the agents and solve the dynamic consensus problem, where the final consensus states can be time varying. In contrast, [2], [4], and [10] adopted a piecewise constant control input to solve the static consensus problem, where the final consensus states are constant. Note that, for single-integrator agents, the event-based controller can be obtained directly from the traditional continuous consensus controller. But to solve the dynamic consensus problem for double-integrator or general linear agents, the continuous controllers cannot be directly implemented in the event-triggered form. Introducing the matrix exponential function $e^{A t}$ in the controller design and the threshold function is an innovative point of our research. The results in [14] dealing with singleand double-integrator dynamics can be regarded a special case of our result. It is worth mentioning that the analysis for convergence and exclusion of the Zeno behavior in our framework is nontrivial and there exist significant challenges.

The rest of this paper is organized as follows. Some useful results and the dynamics are introduced in Section 2. The event-triggered consensus is investigated in Section 3 and the self-triggered scheme is discussed in Section 4. Simulation examples are given in Section 5. Section 6 concludes the paper.

\section{Preliminaries}

\subsection{Notation and graph theory}

Let $\mathbf{R}^{m \times n}$ and $\mathbf{C}^{m \times n}$ be, respectively, the set of $m \times n$ real and complex matrices. Let $\mathbf{1}_{m}$ and $\mathbf{0}_{m}$ denote, respectively, the $m \times 1$ column vector of all ones and all zeros. Let $\mathbf{0}_{m \times n}$ denote the $m \times n$ matrix with all zeros and $I_{m}$ denote the $m \times$ $m$ identity matrix. The superscript $T$ means the transpose for real matrices. We denote by $\lambda_{i}(\cdot)$ the $i$ th eigenvalue of a matrix. By $\operatorname{diag}\left(A_{1}, \cdots, A_{n}\right)$, we denote a block-diagonal matrix with matrices $A_{i}, i=1, \cdots, n$, on its diagonal. A matrix $A \in \mathbf{C}^{m \times m}$ is Hurwitz if all of its eigenvalues have strictly negative real parts. The matrix $A \otimes B$ denotes the Kronecker product of matrices $A$ and $B$. Let $\|\cdot\|$ denote, respectively, the Euclidean norm for vectors and the induced 2-norm for matrices. Let $\|\cdot\|_{F}$ denote the Frobenius norm of a matrix. Let $\operatorname{dim}(\cdot)$ describe the dimension of a square matrix. For a complex number, $\operatorname{Re}(\cdot)$ denotes its real part.

A directed graph $\mathcal{G}$ is a pair $(\mathcal{V}, \mathcal{E})$, where $\mathcal{V}=\left\{v_{1}, \cdots, v_{N}\right\}$ is a nonempty finite set of nodes and $\mathcal{E} \subseteq \mathcal{V} \times \mathcal{V}$ is a set of edges, in which an edge is represented by an ordered pair of distinct nodes. An edge $\left(v_{i}, v_{j}\right)$ means that node $v_{j}$ can receive information from node $v_{i}$ or equivalently node $v_{i}$ can broadcast information to node $v_{j}$. Here we call $v_{i}$ an in-neighbor of $v_{j}$ and $v_{j}$ an out-neighbor of $v_{i}$. A directed 
path from node $v_{i_{1}}$ to node $v_{i_{l}}$ is a sequence of ordered edges of the form $\left(v_{i_{k}}, v_{i_{k+1}}\right), k=1, \cdots, l-1$. A directed graph contains a directed spanning tree if there exists a node called the root such that there exist directed paths from this node to every other node. The adjacency matrix $\mathcal{A}=\left[a_{i j}\right] \in \mathbf{R}^{N \times N}$ associated with the directed graph $\mathcal{G}$ is defined by $a_{i i}=0, a_{i j}>0$ if $\left(v_{j}, v_{i}\right) \in \mathcal{E}$ and $a_{i j}=$ 0 otherwise. The Laplacian matrix $\mathcal{L}=\left[l_{i j}\right] \in \mathbf{R}^{N \times N}$ is defined as $l_{i i}=\sum_{j=1, j \neq i}^{N} a_{i j}$ and $l_{i j}=-a_{i j}, i \neq j$. The graph $\mathcal{G}$ is undirected if $a_{i j}=a_{j i}, \forall i, j=1, \cdots, N$ and directed otherwise.

\subsection{Problem statement and background}

Consider a group of $N$ identical agents with general linear dynamics. The dynamics of the $i$ th agent are described by

$$
\dot{x}_{i}(t)=A x_{i}(t)+B u_{i}(t),
$$

where $x_{i}(t) \in \mathbf{R}^{n}$ is the state, $u_{i}(t) \in \mathbf{R}^{p}$ is the control input, $A \in \mathbf{R}^{n \times n}$, and $B \in \mathbf{R}^{n \times p}$. The communication topology among the agents is represented by a general directed graph $\mathcal{G}$.

The objective of this paper is to design a distributed eventtriggered control law for each agent such that the states of all the agents achieve consensus. We need the following assumption and lemmas to derive our main results.

Assumption 1: The matrix pair $(A, B)$ in (1) is stabilizable and the directed graph $\mathcal{G}$ contains a directed spanning tree.

Lemma 1:[13] If $\mathcal{G}$ contains a directed spanning tree, 0 is a simple eigenvalue of the Laplacian matrix $\mathcal{L}$ and all the other eigenvalues have positive real parts. Moreover $\mathbf{1}_{N}$ is a right eigenvector associated with the zero eigenvalue and there is also a nonnegative left eigenvector $\mathbf{r}=\left[r_{1}, \cdots, r_{N}\right]^{T}$ satisfying $\sum_{j=1}^{N} r_{j}=1$ associated with the zero eigenvalue.

Lemma 2: Suppose that $A \in \mathbf{R}^{n \times n}$ is Hurwitz. Then, for all $t \geq 0$, it holds that $\left\|e^{A t}\right\| \leq\left\|P_{A}\right\|\left\|P_{A}^{-1}\right\| c_{A} e^{a_{A} t}$, where $P_{A}$ is a nonsingular matrix such that $P_{A}^{-1} A P_{A}=J_{A}$ with $J_{A}$ being the Jordan canonical form of $A, c_{A}>0$ is a positive constant determined by $A$, and $\max _{i} \operatorname{Re}\left(\lambda_{i}(A)\right)<a_{A}<0$.

\section{Proof: See Appendix A.}

\section{Event-Triggered Consensus Control}

In this section, we will propose an event-triggered scheme for MAS with general linear dynamics based on state feedback under a general directed graph and prove that no Zeno behavior is exhibited.

The widely-studied consensus controller for (1) was proposed in [9] as

$$
\left.u_{i}(t)=c K \sum_{j=1}^{N} a_{i j}\left(x_{i}(t)-x_{j}(t)\right)\right)
$$

where $c>0$ is the coupling gain, $K \in \mathbf{R}^{p \times n}$ is the feedback gain matrix to be determined, and $a_{i j}$ is the $i j$ th entry of the adjacency matrix $\mathcal{A}$. It was proved in [9] that under the assumption that the directed graph $\mathcal{G}$ contains a directed spanning tree, the controller (2) solves the consensus problem if and only if all matrices $A+c \lambda_{i}(\mathcal{L}) B K$, where $\lambda_{i}(\mathcal{L}) \neq 0$, are Hurwitz.

In (2) each agent needs to use its in-neighbors' states all the time. Thus continuous communication is needed. In order to reduce the communication cost among the agents, we propose an ETCC that only relies on intermittent communication as

$$
u_{i}(t)=c K \sum_{j=1}^{N} a_{i j}\left(e^{A\left(t-t_{k_{i}}^{i}\right)} x_{i}\left(t_{k_{i}}^{i}\right)-e^{A\left(t-t_{k_{j}}^{j}\right)} x_{j}\left(t_{k_{j}}^{j}\right)\right),
$$

where $c, K$, and $a_{i j}$ are defined as in (2), $t_{k_{i}}^{i}$ is the most recent triggering instant of agent $i, k_{i}=1,2, \cdots, A$ is the system matrix of the agents' dynamics, and $x_{i}\left(t_{k_{i}}^{i}\right)$ is the last broadcast state of agent $i$.

For each agent $i$ and $t \geq 0$, we define the state-based measurement error

$$
e_{i}(t)=e^{A\left(t-t_{k_{i}}^{i}\right)} x_{i}\left(t_{k_{i}}^{i}\right)-x_{i}(t) .
$$

The triggering function for each agent $i$ is given by

$$
f_{i}\left(t, e_{i}(t)\right)=\left\|e_{i}(t)\right\|-c_{1} e^{-\alpha t}
$$

where $c_{1}>0$ and $\alpha$ is a positive constant to be determined. Under the ETCC, the controller of agent $i$ monitors its own state continuously. When the state-based measurement error of agent $i$ exceeds a certain given threshold, that is, $f_{i}\left(t, e_{i}(t)\right) \geq 0$, an event is triggered for agent $i$. Agent $i$ updates its controller using its current state and broadcast its current state to its out-neighbors at the same time. Meanwhile, the state-based measurement error of agent $i$ is reset to zero. When agent $i$ receives new states broadcast by its in-neighbors (equivalently, its in-neighbors' events are triggered), the agent also updates its controller immediately. If the state-based measurement error is less than the threshold, there is no communication needed until the next event is triggered.

Without loss of generality, we assume that the latest triggered event occurred at agent $i$ and denote $t^{*}=t_{k_{i}}^{i}$ as the latest triggering instant. That is, there is no event triggered from $t^{*}$ to $t$ for all agents. With the stack vectors $x(t)=\left[x_{1}^{T}(t), \cdots, x_{N}^{T}(t)\right]^{T}, x\left(t^{*}\right)=\left[x_{1}^{T}\left(t^{*}\right), \cdots, x_{N}^{T}\left(t^{*}\right)\right]^{T}$, and $e(t)=\left[e_{1}^{T}(t), \cdots, e_{N}^{T}(t)\right]^{T}$, the closed-loop system of (1) using (3) can be written as

$$
\begin{aligned}
\dot{x}(t) & =\left(I_{N} \otimes A\right) x(t)+(c \mathcal{L} \otimes B K) e^{\left(I_{N} \otimes A\right)\left(t-t^{*}\right)} x\left(t^{*}\right) \\
& =\left(I_{N} \otimes A+c \mathcal{L} \otimes B K\right) x(t)+(c \mathcal{L} \otimes B K) e(t) .
\end{aligned}
$$

Since Assumption 1 holds, it follows from Lemma 1 that there exist matrices $T=\left[\begin{array}{ll}\mathbf{1}_{N} & Y\end{array}\right]$ and $T^{-1}=\left[\begin{array}{r}\mathbf{r} W^{T}\end{array}\right]^{T}$, where $Y \in$ $\mathbf{C}^{N \times(N-1)}$ and $W \in \mathbf{C}^{(N-1) \times N}$, such that $T^{-1} \mathcal{L} T=J_{\mathcal{L}}=$ 
$\left[0, \mathbf{0}_{1 \times(N-1)} ; \mathbf{0}_{N-1}, \Delta\right.$, where $J_{\mathcal{L}}$ is the Jordan canonical form of the matrix $\mathcal{L}$ and $\Delta \in \mathbf{C}^{(N-1) \times(N-1)}$ is a block diagonal matrix. Motivated by [9], we define the disagreement vector $\delta(t)=x(t)-\left(\mathbf{1}_{N} \mathbf{r}^{T} \otimes I_{n}\right) x(t)$ and a new vector

$$
\varepsilon(t)=\left(T^{-1} \otimes I_{n}\right) \delta(t)=\left[\varepsilon_{1}^{T}(t), \varepsilon_{2-N}^{T}(t)\right]^{T},
$$

where $\varepsilon_{1}(t) \in \mathbf{C}^{n}$ and $\varepsilon_{2-N}(t) \in \mathbf{C}^{(N-1) n}$. Using similar derivations to (8)-(12) in [9], the consensus problem for general linear agents can be converted to the stability problem of $\delta(t)$ or $\varepsilon(t)$ under the ETCC (3). Also it follows that $\varepsilon_{1}(t) \equiv \mathbf{0}_{n}$ and the vector $\varepsilon_{2-N}(t)$ satisfies

$$
\dot{\varepsilon}_{2-N}(t)=\Pi \varepsilon_{2-N}(t)+(c \Delta W \otimes B K) e_{2-N}(t),
$$

where $\Pi \triangleq I_{N-1} \otimes A+c \Delta \otimes B K \in \mathbf{C}^{(N-1) n \times(N-1) n}$ and $e_{2-N}(t) \triangleq\left[e_{2}^{T}(t), \cdots, e_{N}^{T}(t)\right]^{T}$.

Remark 1: The stability problem of $\delta(t)$ without the term $e(t)$ was studied in [9]. Compared with continuous controller$\mathrm{s}$ in [9], the ETCC in our paper is switched at the triggering instants, when the state-based measurement error is reset to zero. The main challenge is to exclude the Zeno behavior. Although a non-Zeno analysis has been shown in [14], it is only for integrator agents and undirected communication topologies. The exclusion of the Zeno behavior for MAS with general linear dynamics under general directed graphs is more difficult. We use the property of the matrix exponential function to exclude the Zeno behavior and the corresponding analysis procedure is more challenging than [14].

Now we are ready to present our main result.

Theorem 1: Consider the MAS (1) satisfying Assumption 1. Suppose the triggering function (5) with $c_{1}>0$ and $0<\alpha<-\max \operatorname{Re}\left(\lambda_{i}(\Pi)\right)$, where $\Pi$ is defined after (8). Then, with the ETCC (3) and the triggering function (5), the disagreement vector $\delta(t)$ of the closed-loop system (6) asymptotically converges to zero for all initial conditions if and only if all matrices $A+c \lambda_{i}(\mathcal{L}) B K$, where $\lambda_{i}(\mathcal{L}) \neq 0$, are Hurwitz. Moreover, the closed-loop system (6) does not exhibit the Zeno behavior under the ETCC.

Proof: (Sufficiency) Let $P \in \mathbf{C}^{(N-1) n \times(N-1) n}$ and $P^{-1} \in$ $\mathbf{C}^{(N-1) n \times(N-1) n}$ be the matrices such that $P^{-1} \Pi P=J_{\Pi}$, where $J_{\Pi}$ is the Jordan canonical form of the matrix $\Pi$. From the definition of $\Pi$ after (8), it is obvious that if all matrices $A+c \lambda_{i}(\mathcal{L}) B K$, where $\lambda_{i}(\mathcal{L}) \neq 0$, are Hurwitz, the matrix $\Pi$ is surely Hurwitz and all $\operatorname{Re}\left(\lambda_{i}(\Pi)\right)<0$. Since the triggering function $f_{i}\left(t, e_{i}(t)\right)$ for agent $i$ is reset to zero when an event is triggered. Before the next event is triggered, $f_{i}\left(t, e_{i}(t)\right)$ will not cross zero, that is, $\left\|e_{i}(t)\right\|<c_{1} e^{-\alpha t}$ is satisfied until the next event is triggered. Hence $\left\|e_{2-N}(t)\right\|<\sqrt{N-1} c_{1} e^{-\alpha t}$ and $\left\|e_{2-N}(t)\right\| \rightarrow 0$, as $t \rightarrow \infty$. It follows from (8) and the input-to-state stability argument that $\varepsilon_{2-N}(t)$ approaches zero. Then, it follows that the disagreement vector $\delta(t)$ of the closed-loop system (6) asymptotically converges to zero for all initial conditions, that is, the ETCC (3) solves the event-triggered consensus problem.

Next, we will show that under the ETCC (3), the closed-loop system (6) does not exhibit the Zeno behavior. The solution of $\varepsilon_{2-N}(t)$ can be obtained as

$$
\begin{aligned}
\varepsilon_{2-N}(t)= & e^{\Pi t} \varepsilon_{2-N}(0) \\
& +\int_{0}^{t} e^{\Pi(t-s)}(c \Delta W \otimes B K) e_{2-N}(s) \mathrm{d} s .
\end{aligned}
$$

It follows from Lemma 2 that for $0 \leq s \leq t$,

$$
\begin{aligned}
& \left\|e^{\Pi(t-s)}(c \Delta W \otimes B K) e_{2-N}(s)\right\| \\
\leq & c_{\Pi} c_{1} \sqrt{N-1}((N-1) n-1)\|P\|\left\|P^{-1}\right\|\|c \Delta W \otimes B K\| \\
& \times e^{a_{\Pi}(t-s)} e^{-\alpha s}
\end{aligned}
$$

where $c_{\Pi}$ is a positive constant with respect to $\Pi$ and $\max _{i} \operatorname{Re}\left(\lambda_{i}(\Pi)\right)<a_{\Pi}<0$.

Let $a_{1}=c_{\Pi}((N-1) n-1)\|P\|\left\|P^{-1}\right\|\left\|\varepsilon_{2-N}(0)\right\|$ and $a_{2}=$ $c_{\Pi} c_{1} \sqrt{N-1}((N-1) n-1)\|P\|\left\|P^{-1}\right\|\|c \Delta W \otimes B K\|$. It follows from (9), (10), and Lemma 2 that $\|\varepsilon(t)\|=\left\|\varepsilon_{2-N}(t)\right\| \leq$ $\left(a_{1}+a_{2} /\left|a_{\Pi}+\alpha\right|\right) e^{a_{\Pi} t}+a_{2} /\left|a_{\Pi}+\alpha\right| e^{-\alpha t}$. Then it follows from (7) that $\|\delta(t)\|$ satisfies $\|\delta(t)\| \leq\left\|T \otimes I_{n}\right\|\|\varepsilon(t)\| \leq k_{1} e^{a_{\Pi}{ }^{t}}+$ $k_{2} e^{-\alpha t}, k_{1}=\|T\|\left(a_{1}+a_{2} /\left|a_{\Pi}+\alpha\right|\right)$ and $k_{2}=\|T\| a_{2} /\left|a_{\Pi}+\alpha\right|$.

Let $u(t)$ be the column stack vector of $u_{i}(t)$. Using the property of the Laplacian matrix: $\mathcal{L} \mathbf{1}_{N} \equiv \mathbf{0}_{N}$, we conclude that $\left(I_{N} \otimes B\right) u(t)=(c \mathcal{L} \otimes B K)(\delta(t)+e(t))$. Similarly, $\left\|\left(I_{N} \otimes B\right) u(t)\right\|$ is upper bounded by

$$
\begin{aligned}
\left\|\left(I_{N} \otimes B\right) u(t)\right\| & \leq\|c \mathcal{L} \otimes B K\|(\|\delta(t)\|+\|e(t)\|) \\
& =b_{1} e^{a_{\Pi} t}+b_{2} e^{-\alpha t}
\end{aligned}
$$

where $b_{1}=\|c \mathcal{L} \otimes B K\| k_{1}$ and $b_{2}=\|c \mathcal{L} \otimes B K\|\left(k_{2}+\sqrt{N} c_{1}\right)$.

Recall that we assume that agent $i$ is triggered at the latest triggering instant $t^{*}$. Hence the value $x_{i}\left(t_{k_{i}}^{i}\right)$ remains constant from $t_{k_{i}}^{i}$ to $t^{*}$. It follows from (4) that $\dot{e}_{i}(t)=$ $A e^{A\left(t-t^{*}\right)} x_{i}\left(t^{*}\right)-A x_{i}(t)-B u_{i}(t)=A e_{i}(t)-B u_{i}(t)$. Moreover, with the fact that $\left\|e_{i}(t)\right\| \leq c_{1} e^{-\alpha t}$ before the next event is triggered, $\left\|u_{i}(t)\right\| \leq\|u(t)\|$, and (11), we can get the upper bound of $\left\|\dot{e}_{i}(t)\right\|$ between the two triggered events for agent $i$ as $\left\|\dot{e}_{i}(t)\right\| \leq\|A\|\left\|e_{i}(t)\right\|+\left\|B u_{i}(t)\right\| \leq$ $b_{1} e^{a_{\Pi} t}+d_{2} e^{-\alpha t} \triangleq g(t)$, where $d_{2}=\|A\| c_{1}+b_{2}$. Note that $b_{1}$ and $d_{2}$ are both positive constants here.

Since the latest triggering instant, it follows that $\left\|e_{i}(t)\right\|=$ $\left\|\int_{t^{*}}^{t} \dot{e}_{i}(s) \mathrm{d} s\right\| \leq \int_{t^{*}}^{t} g(s) \mathrm{d} s$. From the definition of the triggering function (5), we know that the next event of agent $i$ will not be triggered before $f_{i}\left(t, e_{i}(t)\right)=0$ or equivalently $\left\|e_{i}(t)\right\|=c_{1} e^{-\alpha t}$. Hence the next event will not be triggered before $\int_{t^{*}}^{t} g(s) \mathrm{d} s=c_{1} e^{-\alpha t}$. Since $t \geq t^{*}$ and both $a_{\Pi}$ and $-\alpha$ are negative, we have $e^{a_{\Pi} t} \leq e^{a_{\Pi} t^{*}}$ and $e^{-\alpha t} \leq e^{-\alpha t^{*}}$. Let $\tau=t-t^{*}$ be the time-interval between the two triggered events. So $\tau$ is greater than or equal to the solution of the implicit equation $\left(b_{1} e^{a_{\Pi} t^{*}}+d_{2} e^{-\alpha t^{*}}\right) \tilde{\tau}=\sqrt{N} c_{1} e^{-\alpha\left(t^{*}+\tilde{\tau}\right)}$, which is equivalent to $\left(b_{1} e^{\left(\alpha+a_{\Pi}\right) t^{*}}+d_{2}\right) \tilde{\tau}=\sqrt{N} c_{1} e^{-\alpha \tilde{\tau}}$. Because $\alpha<-\max _{i} \operatorname{Re}\left(\lambda_{i}(\Pi)\right)$, there must exist a negative 
constant $a_{\Pi}$ such that $\max _{i} \operatorname{Re}\left(\lambda_{i}(\Pi)\right)<a_{\Pi}<-\alpha<0$. As $\alpha<-a_{\Pi}$, we know the term $b_{1} e^{\left.\left(\alpha+a_{\Pi}\right)\right) t^{*}}+d_{2}$ is upper bounded by $b_{1}+d_{2}$. So the solution of the implicit equation is greater than or equal to the solution of $\left(b_{1}+d_{2}\right) \bar{\tau}=$ $\sqrt{N} c_{1} e^{-\alpha \bar{\tau}}$, which is strictly positive. It means that if the coefficients $c_{1}$ and $\alpha$ in (5) satisfy $c_{1}>0$ and $0<\alpha<$ $-\operatorname{Re}\left(\lambda_{1}(\Pi)\right)$, there is a positive lower bound $\bar{\tau}$ on the interevent times for agent $i$. So, the event-triggered consensus problem of the general linear MAS is solved with no Zeno behavior exhibited.

(Necessity) The necessity is obvious. Note that the initial state-based measurement error might not be zero. If at least one matrix $A+c \lambda_{i}(\mathcal{L}) B K$ is not Hurwitz, where $\lambda_{i}(\mathcal{L}) \neq 0$, $\varepsilon_{2-N}(t)$ will go to infinity as $t \rightarrow \infty$ and so will $\delta(t)$. Then, the states of the $N$ agents will not reach consensus for all initial conditions.

Remark 2: Theorem 1 gives a necessary and sufficient condition for the ETCC problem of MAS with general linear dynamics. We extended the existing works [2], [4], [10], and [14] for integrator dynamics to the more general case with general linear dynamics. Other works on the ETCC for general linear systems can be found in [6], [18], [19], and [20]. However, they all have some limitations. In [18], [19], and [20], each agent needed continuous monitoring of its in-neighbors' states and hence continuous communication. The triggering function in [6] and [20] contained a positive constant which could only result in the ultimate boundedness of the final consensus error. In [19], the communication topology was assumed to be undirected and the agents' dynamics are restricted to be stable or neutrally stable. In this paper, we removed all the above limitations by introducing $e^{A t}$ in the controller and a design parameter $\alpha$ in the triggering condition. The tradeoff here is that the computation load is increased in the controller.

Remark 3: The design parameter $\alpha$ in (5) plays an important role in the convergence rate of the measurement error vector. It is noted from (9) that $-\operatorname{Re}\left(\lambda_{i}(\Pi)\right)$ could be understood as the convergence rate of the closed-loop system (6). Actually, the convergence rate $\alpha$ of the measurement error's threshold should be smaller than the convergence rate $-\operatorname{Re}\left(\lambda_{i}(\Pi)\right)$ of (6). Otherwise, there might the Zero behavior.

Remark 4: Using the event-triggered scheme, there is a tradeoff between the communication load and the convergence speed. According to practical needs, an appropriate design parameter $\alpha$ in (5) can be selected to achieve the tradeoff. For example, if this parameter is smaller, then the convergence is faster but more frequent communication is needed. Otherwise, the convergence is slower, but less frequent communication is needed.

Motivated by [9], we now present a multi-step event-triggered consensus control algorithm. Algorithm 1: Given $(A, B)$ that is stabilizable, an event-triggered algorithm in the form of (3) and (5) solving the consensus problem of general linear MAS can be constructed according to the following steps.

(1) Solve the following linear matrix inequality

$$
A P+P A^{T}-2 B B^{T}<0
$$

to get one symmetric positive-definite solution $P$. Then, choose the feedback gain matrix $K=-B^{T} P^{-1}$.

(2) Select the coupling gain $c$ in (3) given by $c>\frac{1}{\min \operatorname{Re}\left(\lambda_{i}(\mathcal{L})\right)}$, where $\lambda_{i}(\mathcal{L}) \neq 0$, denote the nonzero eigenvalues of Laplacian matrix $\mathcal{L}$.

(3) Choose the constants in the function (5) to satisfy $c_{1}>0$ and $0<\alpha<-\max _{i} \operatorname{Re}\left(\lambda_{i}(\Pi)\right)$, where $\Pi$ is defined after $(8)$.

The steps 1 and 2 are borrowed from [9] to ensure that $A+c \lambda_{i}(\mathcal{L}) B K, i=2, \cdots, N$ are Hurwitz. Since Assumption 1 holds, there must exist a matrix $P$ satisfying (12).

Remark 5: Global information is needed to determine the exact upper or lower bound of the parameters but not the parameters themselves. Algorithm 1 itself is still distributed as it uses local (neighbor-to-neighbor) communication. In applications, we can select the parameters more conservatively as long as they satisfy their bounds. One idea is to determine the parameters offline using the worst case graph topology before running the algorithm. In addition, there exist some distributed algorithms estimating the eigenvalues of a Laplacian matrix [5], which could be adopted to estimate the upper or lower bound of the parameters in this paper.

If the agents' states are not measurable in practice, we could use some observer-based method to implement an ETCC for MAS described by

$\dot{x}_{i}(t)=A x_{i}(t)+B u_{i}(t), y_{i}(t)=C x_{i}(t)$,

where $x_{i}(t), u_{i}(t), A$, and $B$ are defined in (1), $y_{i}(t) \in \mathbf{R}^{q}$ is the measured output, and $C \in \mathbf{R}^{q \times n}$. In this case, we make the following assumption.

Assumption 2: $(A, B, C)$ in (13) is stabilizable and detectable and the directed graph $\mathcal{G}$ contains a directed spanning tree.

The observer-based ETCC for each agent $i$ is given by

$$
\begin{aligned}
\dot{\hat{x}}_{i}(t) & =A \hat{x}_{i}(t)+B u_{i}(t)+F\left(\hat{y}_{i}(t)-y_{i}(t)\right) \\
\hat{y}_{i}(t) & =C \hat{x}_{i}(t) \\
u_{i}(t) & =c K \sum_{j=1}^{N} a_{i j}\left(e^{A\left(t-t_{k_{i}}^{i}\right)} \hat{x}_{i}\left(t_{k_{i}}^{i}\right)-e^{A\left(t-t_{k_{j}}^{j}\right)} \hat{x}_{j}\left(t_{k_{j}}^{j}\right)\right),
\end{aligned}
$$

where $\hat{x}_{i}(t) \in \mathbf{R}^{n}$ is the observer state, $\hat{y}_{i}(t) \in \mathbf{R}^{q}$ is the output of the observer, $F$ is the feedback gain matrix to be determined, $c, K$, and $a_{i j}$ are defined in (2), and $t_{k_{i}}^{i}$ is defined in (3). We use $\hat{x}_{i}\left(t_{k_{i}}^{i}\right)$ to denote the last broadcast observer state of agent $i$. We define the observer-based measurement error $e_{i}(t)=e^{A\left(t-t_{k_{i}}^{i}\right)} \hat{x}_{i}\left(t_{k}^{i}\right)-\hat{x}_{i}(t)$. The triggering function for each agent is defined the same as (5). Then, we have the following result. The proof is omitted due to space limitation.

Theorem 2: Consider the MAS (13) satisfying Assumption 2. Suppose the triggering function (5) with $c_{1}>0$ and $0<\alpha<-\max \operatorname{Re}\left(\lambda_{i}(\bar{\Pi})\right)$, where $\bar{\Pi} \triangleq\left[I_{N-1} \otimes A+c \Delta \otimes\right.$ $\left.B K, c \Delta \otimes B \stackrel{i}{K} ; \mathbf{0}_{(N-1) n \times(N-1) n}, I_{N-1} \otimes(A+F C)\right]$. Then, with the observer-based ETCC (14) and the triggering function (5), the disagreement vector of the closed-loop system 
asymptotically converges to zero for all initial conditions if and only if all matrices $A+c \lambda_{i}(\mathcal{L}) B K$, where $\lambda_{i}(\mathcal{L}) \neq 0$, and $A+F C$ are Hurwitz. Moreover, the closed-loop system does not exhibit Zeno behavior under the observer-based ETCC.

\section{Self-Triggered Consensus Control}

The event-triggered scheme proposed in the last section needs each agent to monitor its own states continuously to check the triggering function. In this section, we extend the result to the self-triggered scheme, where the continuous selfstate monitoring is relaxed. The next triggering instant $t_{k_{i}+1}^{i}$ for agent $i$ is preset at the previous triggering instant $t_{k_{i}}^{i}$. No monitoring is required between two triggered events.

For agent $i$, since the $k_{i}$ th triggering instant $t_{k_{i}}^{i}$, it$\mathrm{s}$ state can be calculated by $x_{i}(t)=e^{A\left(t-t_{k_{i}}^{i}\right)} x_{i}\left(t_{k_{i}}^{i}\right)+$ $\int_{t_{k_{i}}^{i}}^{t} e^{A(t-s)} B u_{i}(s) \mathrm{d} s$ with $u_{i}(s)=c K \sum_{j=1}^{N} a_{i j}\left(e^{A\left(s-t_{k_{i}}^{i}\right)} x_{i}\left(t_{k_{i}}^{i}\right)-\right.$ $\left.e^{A\left(s-t_{k_{j}}^{j}\right)} x_{j}\left(t_{k_{j}}^{j}\right)\right)$. Note that $e_{i}(t)=e^{A\left(t-t_{k_{i}}^{i}\right)} x_{i}\left(t_{k}^{i}\right)-x_{i}(t)=$ $-\int_{t_{k_{i}}^{i}}^{t} e^{A(t-s)} B u_{i}(s) \mathrm{d} s$ and $t_{k_{i}}^{i}, x_{i}\left(t_{k_{i}}^{i}\right), t_{k_{j}}^{j}$, and $x_{j}\left(t_{k_{j}}^{j}\right)$ are known to agent $i$ at the triggering instant $t_{k_{i}}^{i}$. Recalling (5) and using the notation $\xi_{i}=t-t_{k_{i}}^{i}$, the self-triggering function is written as

$$
\begin{aligned}
f_{i}\left(t, e_{i}(t)\right) & =\left\|\int_{t_{k_{i}}^{i}}^{t_{k_{i}}^{i}+\xi_{i}} e^{A\left(t_{k_{i}}^{i}+\xi_{i}-s\right)} B u_{i}(s) \mathrm{d} s\right\|-c_{1} e^{-\alpha\left(t_{k_{i}}^{i}+\xi_{i}\right)} \\
& =0
\end{aligned}
$$

where $\xi_{i}$ can be decided by agent $i$ at time $t_{k_{i}}^{i}$.

Based on the above observation, the self-triggering policy to determine the next triggering instant for agent $i$ at time $t_{k_{i}}^{i}$ is defined as follows. Assume the solution of the implicit equation (15) is $\bar{\xi}_{i}$. Then the next triggering instant $t_{k_{i}+1}^{i}$ takes place at most $\bar{\xi}_{i}$ time units after $t_{k_{i}}^{i}$, i.e., $t_{k_{i}+1}^{i} \leq t_{k_{i}}^{i}+\bar{\xi}_{i}$. For agent $i$ and all $t \in\left[t_{k_{i}}^{i}, t_{k_{i}}^{i}+\bar{\xi}_{i}\right]$, if there is an event triggered in one of its in-neighbors, i.e., some new state is broadcast to agent $i$, agent $i$ re-check the self-triggering function (15) with the new information. Otherwise, agent $i$ waits until its preset triggering instant $t_{k_{i}+1}^{i}$ to re-compute the condition (15). Similar to the last section, the time interval between two consecutive triggered events is strictly positive for agent $i$. Note that there is no continuous monitoring required for each agent. The triggering instant is preset by agent $i$ itself at the previous triggering instant. Now we present a multistep STCC algorithm.

Algorithm 2: Given $(A, B)$ that is stabilizable, a self-trigger based algorithm in the form of (3) and (15) solving the consensus problem of general linear MAS can be constructed according to the following steps.

(1) and (2) Same as Algorithm 1.

(3) Choose the constants in the function (15) to satisfy $c_{1}>0$ and $0<\alpha<-\max \operatorname{Re}\left(\lambda_{i}(\Pi)\right)$, where $\Pi$ is defined after (8).

(4) At time $t_{k_{i}}^{i}$, solve the self-triggering function (15) to get a solution $\bar{\xi}_{i}$.

(5) Preset the next triggering instant at $t_{k_{i}+1}^{i}$ which is at most $\bar{\xi}_{i}$ after $t_{k_{i}}^{i}$, i.e., $t_{k_{i}+1}^{i} \leq t_{k_{i}}^{i}+\bar{\xi}_{i}$.

(6) For all $t \in\left[t_{k_{i}}^{i}, t_{k_{i}}^{i}+\bar{\xi}_{i}\right]$, if there is an event triggered in one of its in-neighbors, re-check (15) using the new information received. Otherwise, agent $i$ waits until $t_{k_{i}+1}^{i}$.

The preceding analysis, along with Theorem 1, yields the following result.

Theorem 3: Consider the MAS (1) satisfying Assumption 1. Suppose that in the self-triggering function (15) $c_{1}>0$ and $0<\alpha<-\max \operatorname{Re}\left(\lambda_{i}(\Pi)\right)$, where $\Pi$ is defined after (8). Assume that the next triggering instant is chosen according to Algorithm 2. Then, with the controller (3), the disagreemen$\mathrm{t}$ vector $\delta(t)$ of the closed-loop system (6) asymptotically converges to zero for all initial conditions if and only if al1 matrices $A+c \lambda_{i}(\mathcal{L}) B K$, where $\lambda_{i}(\mathcal{L}) \neq 0$, are Hurwitz. Moreover, the closed-loop system (6) does not exhibit the Zeno behavior.

Remark 6: Theorem 3 solves the self-triggered consensus problem of MAS with general linear dynamics. Compared with the key result in the last section, each agent does not need its own continuous state measurement and can preset the next triggering instant at the previous event. The STCC relaxes the requirement of continuous self-state monitoring between the controller updates.

\section{Simulation}

In this section, we illustrate the above theoretical results by simulation. Consider a group of 6 agents with general linear dynamics described by (1), where $A=[0,1 ;-1 ; 0]$ and $B=[1 ; 1]$. We choose the feedback gain matrix $K=\left[\begin{array}{ll}-2-1 & -1\end{array}\right.$ so that $A+B K$ is Hurwitz. The communication topology among the agents is shown in Fig. 1, which is a directed graph containing a directed spanning tree. The Laplacian matrix of the communication graph is $\mathcal{L}=[3,0,0,-1,-1,-1 ;-1,1,0,0,0,0 ;-1,-1,2,0,0,0 ;-1$, $0,0,1,0,0 ; 0,0,0-1,1,0 ; 0,0,0,0,-1,1]$. Obviously, the nonzero eigenvalues of the Laplacian matrix $\mathcal{L}$ are $1,1.3376 \pm 0.5623 i, 2,3.3247$. We just illustrate the ETCC algorithm (Algorithm 1). Here by step 2 of Algorithm 1, we choose $c=1.1$. The eigenvalues of $\Pi$ defined after (8) are $-0.4423,-0.5269,-0.5729 \pm 0.136 i,-0.861,-2.439$, $-3.8413 \pm 1.9915 i,-6.0731$, and -10.5293 . So, according to the conditions required in step 3 of Algorithm 1, we choose $c_{1}=0.6$ and $\alpha=0.4$. The initial states are given by $x_{1}(0)=[0.4 ; 0.3], x_{2}(0)=[0.5 ; 0.2], x_{3}(0)=[0.6 ; 0.1]$, $x_{4}(0)=[0.7 ; 0], x_{5}(0)=[0.8 ;-0.1]$, and $x_{6}(0)=[0.4 ;-0.2]$. The state trajectories are presented in Fig. 2, while the trajectories under the controller (2) proposed in [9] are depicted in Fig. 3. From Figs. 2 and 3, we can see the convergent time is almost the same. But the dynamic performance under the ETCC is a little worse than the controller (2). This is due to the tradeoff between performance and communication cost. The state-based measurement errors and their thresholds of agents are shown in Fig. 4. The bottom of Fig. 2 presents the triggering instants of Algorithm 1, where we can see that the communication among agents is discrete and the 


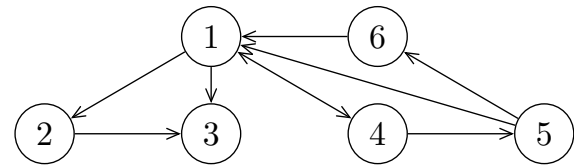

Fig. 1. The communication graph among the agents.
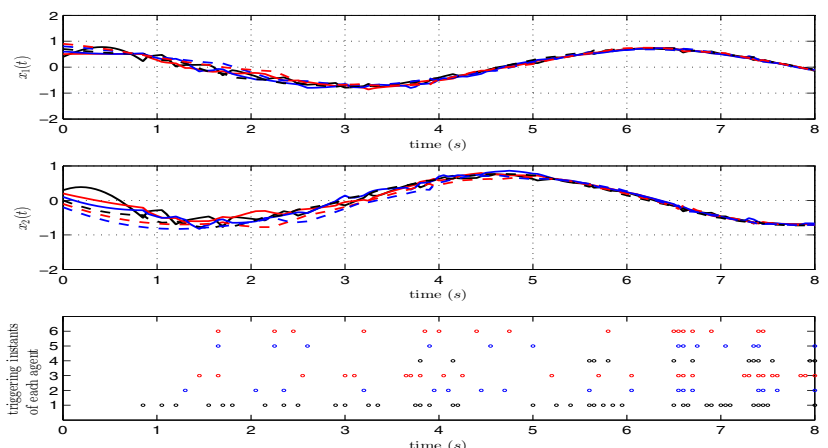

Fig. 2. The states and the triggering instants of each agent under the ETCC.
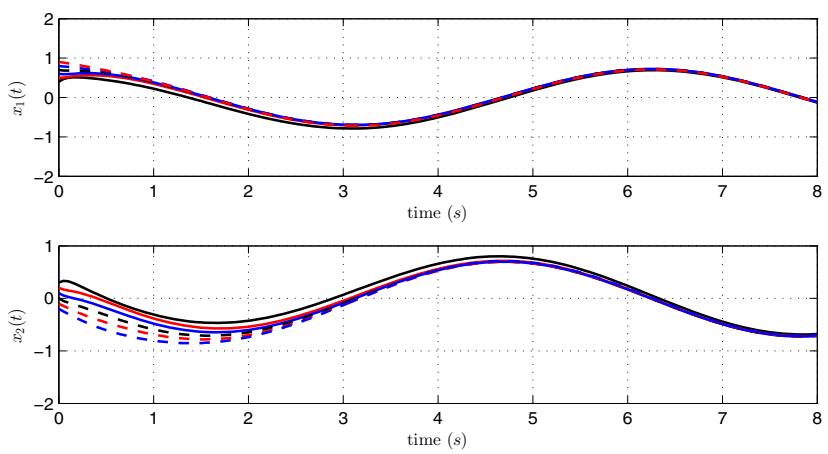

Fig. 3. The states of each agent under the traditional controller.
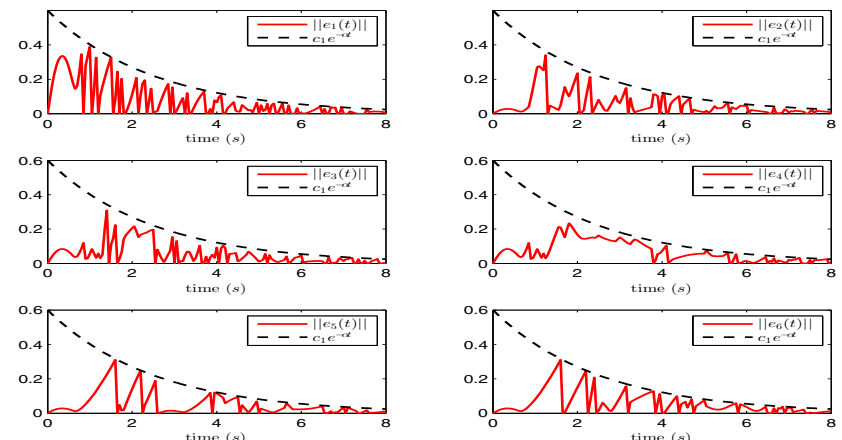

Fig. 4. The errors and the thresholds of the errors of each agent under the ETCC.

Zeno behavior is avoided. Compared with the continuous communication, the ETCC can reduce the communication cost and save much communication source.

\section{Conclusion}

This paper considered the event-triggered consensus problem for MAS with general linear dynamics under general directed graphs. We proposed a decentralized event-triggered broadcasting algorithm for each agent to achieve consensus based on state feedback, without requiring continuous communication among agents. It is proved that under the proposed event-triggered control algorithm implemented in multiple steps, there is no Zeno behavior exhibited. We further proposed a self-triggered control algorithm to relax the requirement of continuous self-monitoring for each agent, under which the next triggering instant is preset by each agent itself at the previous triggering instant. This paper extended the existing results on event-triggered consensus control without continuous communication for single-integrator and double-integrator systems to the case of agents with general linear dynamics. Delay/switching event-triggered consensus for continuous-time MAS with general linear dynamics and event-triggered consensus for discrete-time linear MAS are future topics to be discussed. It is worth mentioning that the proposed piecewise continuous control input is just a sufficient condition to exclude the Zeno behavior. How to design a piecewise constant control input to solve the dynamic consensus problem is also one of our future research topics.

\section{Appendix}

\section{A Proof of Lemma 2}

For every $A$ with $s$ distinct eigenvalues $\left\{\lambda_{1}(A), \cdots, \lambda_{s}(A)\right\}$, there is a nonsingular matrix $P_{A}$ such that $P_{A}^{-1} A P_{A}=J_{A}=$ $\operatorname{diag}\left\{J_{1}, \cdots, J_{s}\right\}$ and $\sum_{i=1}^{s} \operatorname{dim}\left(J_{i}\right)=n$. For each eigenvalue $\lambda_{i}(A), i=1, \cdots, s$, the Jordan segment $J_{i}$ is made up of $m_{i}$ Jordan blocks, that is, $J_{i}=\operatorname{diag}\left\{J_{i 1}, \cdots, J_{i m_{i}}\right\}$ with

$J_{i j}=\left[\begin{array}{cccc}\lambda_{i}(A) & 1 & \cdots & 0 \\ \vdots & \ddots & \ddots & \vdots \\ 0 & \cdots & \lambda_{i}(A) & 1 \\ 0 & \cdots & 0 & \lambda_{i}(A)\end{array}\right]$

$i=1, \cdots, s, j=1, \cdots, m_{i}$, and $\sum_{j=1}^{m_{i}} \operatorname{dim}\left(J_{i j}\right)=\operatorname{dim}\left(J_{i}\right)$.

Note that the matrix exponential function $e^{J_{A} t}$ of the matrix $J_{A}$ is of a block diagonal form given by $e^{J_{A} t}=$ $\operatorname{diag}\left\{e^{J_{1} t}, \cdots, e^{J_{s} t}\right\}$ with $e^{J_{i} t}=\operatorname{diag}\left\{e^{J_{i 1} t}, \cdots, e^{J_{i m_{i}} t}\right\}$. Note that $e^{J_{i j} t}$ has the form

$e^{J_{i j} t}=\left[\begin{array}{cccc}e^{\lambda_{i}(A) t} & t e^{\lambda_{i}(A) t} & \cdots & \frac{t^{\operatorname{dim}\left(J_{i j}\right)-1}}{\left(\operatorname{dim}\left(J_{i j}\right)-1\right) !} e^{\lambda_{i}(A) t} \\ \vdots & \ddots & \ddots & \ddots \\ 0 & \cdots & e^{\lambda_{i}(A) t} & t e^{\lambda_{i}(A) t} \\ 0 & \cdots & 0 & e^{\lambda_{i}(A) t}\end{array}\right]$. 
As the induced 2-norm is always not greater than the Frobenius norm for a matrix, we get

$$
\begin{aligned}
\left\|e^{J_{i j} t}\right\| & \leq\left\|e^{J_{i j} t}\right\|_{F} \leq \varrho\left|e^{\lambda_{i}(A) t}\right| \\
& \leq\left(\operatorname{dim}\left(J_{i j}\right)-1\right) e^{\operatorname{Re}\left(\lambda_{i}(A)\right) t} \max \left\{1, t^{\operatorname{dim}\left(J_{i j}\right)-1}\right\}
\end{aligned}
$$

where $\varrho=\left\|\left[\begin{array}{cccc}1 & t & \cdots & \frac{t^{\operatorname{dim}\left(J_{i j}\right)-1}}{\left(\operatorname{dim}\left(J_{i j}\right)-1\right) !} \\ \vdots & \ddots & \ddots & \vdots \\ 0 & \cdots & 1 & t \\ 0 & \cdots & 0 & 1\end{array}\right]\right\|_{F}$

Similarly, for the matrix exponential function $e^{A t}$ of the ma$\operatorname{trix} A$, it follows that

$$
\begin{aligned}
\left\|e^{A t}\right\| & =\left\|P_{A} e^{J_{A} t} P_{A}^{-1}\right\| \\
& \leq(n-1)\left\|P_{A}\right\|\left\|P_{A}^{-1}\right\| e^{\max \operatorname{Re}\left(\lambda_{i}(A)\right) t} \max _{i, j}\left\{1, t^{\operatorname{dim}\left(J_{i j}\right)-1}\right\} .
\end{aligned}
$$

Since $A$ is Hurwitz, $\operatorname{Re}\left(\lambda_{i}(A)\right)$ are all strictly negative. By noting that $\operatorname{dim}\left(J_{i j}\right) \leq n=\operatorname{dim}(A)$ is finite, there must exist constants $c_{A}>0$ and $\max \operatorname{Re}\left(\lambda_{i}(A)\right)<a_{A}<0$ such that $e^{\max _{i} \operatorname{Re}\left(\lambda_{i}(A)\right) t} \max _{i, j}\left\{1, t^{\operatorname{dim}\left(J_{i j}\right)-1}\right\}<c_{A} e^{a_{A} t}$. Thus, we have that $\left\|e^{A t}\right\| \leq(n-1)\left\|P_{A}\right\|\left\|P_{A}^{-1}\right\| c_{A} e^{a_{A} t}$.

In order to find $c_{A}$ and $a_{A}$ to satisfy the inequality

$$
e^{\max _{i} \operatorname{Re}\left(\lambda_{i}(A)\right) t} \max _{i, j}\left\{1, t^{\operatorname{dim}\left(J_{i j}\right)-1}\right\}<c_{A} e^{a_{A} t}, \forall t>0,
$$

let's consider two cases: $t \leq 1$ and $t>1$. In the following proving process, we define $\lambda \triangleq \max _{i} \operatorname{Re}\left(\lambda_{i}(A)\right)$ and $d \triangleq$ $\operatorname{dim}\left(J_{i j}\right)-1$ for simplicity.

a) For the first case, the inequality becomes $e^{\lambda t}<c_{A} e^{a_{A} t}$. Since $\lambda<0$, we just select $a_{A}$ to satisfy $\lambda<a_{A}<0$ which can be easily realized. To find a proper $c_{A}$, we define a function $f(t)$ respect to $t$ as $f(t) \triangleq c_{A} e^{a_{A} t}-e^{\lambda t}$. Note that if $c_{A}>1$, we always have $f(t)>0, \forall t>0$ since $e^{a_{A} t}>e^{\lambda t}, \forall t>0$.

b) For the second case, the inequality becomes $e^{\lambda t} t^{d}<$ $c_{A} e^{a_{A} t}$. We also select $a_{A}$ such that $\lambda<a_{A}<0$ and define a function $f(t)$ as $f(t) \triangleq c_{A} e^{\left(a_{A}-\lambda\right) t}-t^{d}$. Note that we just need to find a $c_{A}>0$ such that $f(t)>0, \forall t>0$ since $e^{\lambda t}>0, \forall t>0$. The derivative of $f(t)$ is

$f^{\prime}(t)=\left\{\begin{array}{c}c_{A}\left(a_{A}-\lambda\right) e^{\left(a_{A}-\lambda\right) t}-1, d=1 \\ c_{A}\left(a_{A}-\lambda\right) e^{\left(a_{A}-\lambda\right) t}-d t^{d-1}, d>1\end{array}\right.$.

Letting $f^{\prime}(t)=0$, we have

$$
\left\{\begin{array}{c}
c_{A}\left(a_{A}-\lambda\right) e^{\left(a_{A}-\lambda\right) t}=1, d=1 \\
c_{A}\left(a_{A}-\lambda\right) e^{\left(a_{A}-\lambda\right) t}=d t^{d-1}, d>1
\end{array} .\right.
$$

Assume that the solution of the last equation is $\bar{t}$. Subscribing the last equation into $f(t)$, we know that

$f(\bar{t})=\left\{\begin{array}{c}\frac{1}{a_{A}-\lambda}-\bar{t}, d=1 \\ \frac{d \bar{t}^{d-1}}{a_{A}-\lambda}-\bar{t}^{d}, d>1\end{array}\right.$.

Letting $f(\bar{t})=0$, we have $\bar{t}=\frac{d}{a_{A}-\lambda}$. Subscribing this equation into $f(t)$, we will get the sufficient condition of $c_{A}$ to satisfy $f(t)>0, \forall t>0$ is that $c_{A}>\left(\frac{d}{e\left(a_{A}-\lambda\right)}\right)^{d}$.

Until now, we have given the way of finding proper constants $c_{A}$ and $a_{A}$ to satisfy the inequality

$e^{\max _{i} \operatorname{Re}\left(\lambda_{i}(A)\right) t} \max _{i, j}\left\{1, t^{\operatorname{dim}\left(J_{i j}\right)-1}\right\}<c_{A} e^{a_{A} t}, \forall t>0$.

\section{References}

[1] Ceragioli F., Persis C. D., \& Frasca P. (2011). Discontinuities and hysteresis in quantized average consensus. Automatica, 47(9), 1916-1928.

[2] Dimarogonas D. V., Frazzoli E., \& Johansson K. H. (2012). Distributed event-triggered control for multi-agent systems. IEEE Transactions on Automatic Control, 57(5), 1291-1297.

[3] Dimarogonas D. V. \& Johansson K. H. (2009). Eventtriggered control for multi-agent systems. Proc. 48th IEEE CDC, 7131-7136.

[4] Fan Y., Feng G., Wang Y., \& Song C. (2013). Distributed event-triggered control of multi-agent systems with combinational measurements. Automatica, 49(2), 671675.

[5] Franceschelli M., Gasparri A., Giua A., \& Seatzu C. (2013). Decentralized estimation of laplacian eigenvalues in multi-agent systems. Automatica, 49(4), 1031-1036.

[6] Garcia E., Cao Y., Giua A., \& Casbeer D. (2014). Decentralized event-triggered consensus with general linear dynamics. Automatica, 50(10), 2633-2640.

[7] Garcia E., Cao Y., Yu H., Giua A., Antsaklis P., \& Casbeer D. (2013). Decentralised event-triggered cooperative control with limited communication. International Journal of Control, 86(9), 1479-1488.

[8] Jadbabaie A., Lin J., \& Morse A. S. (2013). Coordination of groups of mobile autonomous agents using nearest neighbor rules. IEEE Transactions on Automatic Control, 48(6), 988-1001.

[9] Li Z., Duan Z., Chen G., \& Huang L. (2010). Consensus of multiagent systems and synchronization of complex networks: a unified viewpoint. IEEE Transactions on Circuits and Systems I, 57(1), 213-224.

[10] Meng X. \& Chen T. (2013). Event based agreement protocols for multi-agent networks. Automatica, 49(7), $2125-2132$.

[11] Olfati-Saber R., Fax J. A., \& Murray R. M. (2007). Consensus and cooperation in networked multi-agent systems. Proceedings of the IEEE, 95(1), 215-233. 
[12] Ren W. \& Beard R. (2008). Distributed Consensus in Multi-vehicle Cooperative Control. London: SpringerVerlag.

[13] Ren W. \& Cao Y. (2011). Distributed Coordination of Multi-agent Networks. London: Springer-Verlag.

[14] Seyboth G. S., Dimarogonas D. V., \& Johansson K. H. (2013). Event-based broadcasting for multi-agent average consensus. Automatica, 49(1), 245-252.

[15] Wen G., Duan Z., Yu W., \& Chen G. (2013). Consensus of second-order multi-agent system with delayed nonlinear dynamics and intermittent communications. International Journal of Control, 86(2), 322-331.

[16] Xiao F. \& Wang L. (2008). Asynchronous consensus in continuous-time multi-agent systems with switching topology and time-varying delays. IEEE Transactions on Automatic Control, 53(8), 1804-1816.

[17] Yu W., Chen G., \& Cao M. (2010). Some necessary and sufficient conditions for second-order consensus in multiagent dynamical systems. Automatica, 46(6), 1089-1095.

[18] Zhang H., Feng G., Yan H., \& Chen Q. (2014). Observer-based output feedback event-triggered control for consensus of multi-agent systems. IEEE Transactions on Industrial Electronics, 61(9), 4885-4894.

[19] Zhang Z., Hao F., Zhang L., \& Wang L. (2014). Consensus of linear multi-agent systems via eventtriggered control. International Journal of Control, 87(6), 1243-1251.

[20] Zhu W., Jiang Z., \& Feng G. (2014). Event-based consensus of multi-agent systems with general linear models. Automatica, 50(2), 552-558.

[21] Chen W. \& Ren W. (2016). Event-triggered zerogradient-sum distributed consensus optimization over directed networks. Automatica, 65(3), 90-97.

[22] Yang D., Ren W., \& Liu X. (2014). Decentralized consensus for linear multi-agent systems under general directed graphs based on event-triggered/self-triggered strategy. Proc. 53th IEEE CDC, 1983-1988. 